\title{
Kinematics and CMD of the globular cluster NGC 4147
}

\author{
J.J. Wang ${ }^{1,2,3}$, L. Chen ${ }^{1,2}$, Z.Y. Wu ${ }^{1,2}$, A.C. Gupta ${ }^{4,5,6}$, and M. Geffert ${ }^{7}$ \\ 1 Shanghai Astronomical Observatory, CAS, Shanghai 200030, PR China \\ 2 National Astronomical Observatory, CAS, Beijing 100080, PR China \\ 3 Joint Laboratory for Optical Astronomy, CAS, Shanghai 200030, PR China \\ 4 Department of Physics, University of Gorakhpur, Gorakhpur 273 009, India \\ 5 U. P. State Observatory, Manora Peak, Nainital 263 129, India \\ 6 Physical Research Laboratory, Navarangpura, Ahmedabad 380 009, India \\ 7 Sternwarte der Universität Bonn, Auf dem Hügel 71, D-53121 Bonn, Germany
}

Received May 6; accepted December 9, 1999

\begin{abstract}
Astrometry and BVRI CCD photometry of 115 stars down to $B=17.6$ in the region of about $11^{\prime} \times 11^{\prime}$ around the globular cluster NGC 4147 was performed. In the astrometric reduction, three earlier epoch plates taken at Sheshan, Shanghai, China, in 1958 and four recent epoch $B$-passband CCD frames taken at Kavalur, India, were used. The data were reduced to a catalogue based on measurements of stars on seven plates with an epoch period from 1917 to 1979 taken in Bonn, Germany, and ultimately to the Hipparcos catalogue. The reduction was done with the central overlapping method. Based on the new proper motion data, the membership probabilities of 115 stars were determined. Furthermore, three colourmagnitude diagrams (CMD) of $V$ versus $B-V, V-R$ and $V-I$, respectively, for $\mathrm{HB}$ and GB stars were constructed from CCD photometry obtained with the Vainu Bappu Telescope. An absolute proper motion of the cluster of $-2.08 \pm 0.48 \mathrm{mas} / \mathrm{yr}$ in right ascension and $-3.07 \pm$ $0.46 \mathrm{mas} / \mathrm{yr}$ in declination has been obtained. The space velocity and apogalactic distance of the cluster with respect to the Galactic standard of rest were calculated. In contrast to the first results given in the literature we obtain a significantly lower velocity and a smaller apogalactic distance for NGC 4147.
\end{abstract}

Key words: stars: kinematics — stars: HertzsprungRussell and $\mathrm{C}-\mathrm{M}$ diagrams - globular clusters: individual: NGC 4147

\section{Introduction}

Globular clusters (GCs) are among the oldest stellar objects in the Milky Way. Due to the total luminosity from

Send offprint requests to: L. Chen, e-mail: chenlic@online.sh.cn the large number of stars, they can be observed far away. They play very important rôles in the study of the structure and evolution of the Galaxy. For instance, with GCs as a new constraint, Dauphole \& Colin (1995) studied the mass distribution in our Galaxy, re-calculated the model potential and obtained a better consistency with the observations. The formation of the Galaxy was studied by Eggen et al. (1962; hereafter ELS), which were in favour of a pressure supported rapid collapse model (ELS model), while Searle \& Zinn (1978; hereafter SZ) proposed an accretion model of Galaxy formation (SZ model). Their model is based on the fact that the globular clusters could be considered as relics of some sub-systems merging with the Galaxy, these relics still having different kinematics and metal abundances. Geffert et al. (1995) and Dauphole et al. (1996), using a sample of 26 GCs with absolute proper motion data, found a correlation between the apogalactic distances and the metallicities in support of the ELS model.

Kinematic parameters of globular clusters may provide important information for the understanding of the formation of the Galaxy. The reliability of their determination, however, depends of the quality of the proper motion measuring. In fact, the accuracy of radial velocities of the GCs, at present, is better than $\pm 1 \mathrm{~km} \mathrm{~s}^{-1}$ and the accuracy of distance determination is better than $10 \%$. On the other hand, if the proper motion accuracy were \pm 1 mas $/ y r-a$ typical value for a recent proper motion determinationit would lead to an uncertainty of $50 \mathrm{~km} \mathrm{~s}^{-1}$ for the tangential velocity of an object with a distance of $10 \mathrm{kpc}$. Moreover, proper motions should be linked to an inertial reference system and this correction itself might have larger uncertainties that could result in errors worse than $\pm 1 \mathrm{mas} / \mathrm{yr}$.

To avoid such errors from the corrections, Brosche et al. (1983) have determined the proper motions of 
reference stars in the fields of globular clusters relative to a large number of extragalactic objects, using the Lick plates taken for the NPM program. They so reduced the data directly to an inertial system. Using the above method, Brosche et al. (1985) obtained absolute proper motions of 42 stars in the globular cluster NGC 4147 and found the mean absolute proper motion of $\mu_{\alpha} \cos \delta=$ $-2.7 \pm 0.6 \mathrm{mas} / \mathrm{yr}, \mu_{\delta}=+0.9 \pm 0.6 \mathrm{mas} / \mathrm{yr}$ (in 1991, they recalculated the both errors as $\pm 1.3 \mathrm{mas} / \mathrm{yr}$ ). One problem in their work is, that the number of observed stars is too small and no photometric data are given. Since also the proper motion accuracy of individual stars is poor, membership cannot be estimated reliably and only three field stars in the sample were found. Another problem is that Brosche et al. (1985) used the AGK3 Catalogue to determine the positions of reference stars so that their results still unavoidably contain systematic differences from an inertial system. Now we can fortunately use the Hipparcos Catalogue, which is the best optical realization of the International Celestial Reference System, to minimize such a difference.

The colour-magnitude diagram of the globular cluster NGC 4147 was given earlier by Sandage \& Walker (1955) and was studied by Friel et al. (1987) later. Aurière \& Lauzeral (1991) presented the $B V$ CCD photometry of this cluster and presented a CMD of 532 stars up to $V=21$ within a $100^{\prime \prime} \times 160^{\prime \prime}$ area. In the CMD, they found a red giant $25^{\prime \prime}$ away from the cluster center, which was about 1 mag brighter and 0.4 mag redder than other red giants in the cluster. The giant is located on the extension of the red giant branch (RGB) of the cluster CMD but it may also be a field star. The proper motion data of this star may provide a clarification of its membership.

In the present work more accurate absolute proper motions for more stars in the cluster NGC 4147 region are determined. With the proper motion result, the membership probabilities of the stars are estimated using the maximum likelihood method. Meanwhile, multi-colour photometry was made of stars in the cluster. The proper motion membership probability is used to purify the CMDs. Then, with this member sample, a more reliable mean absolute proper motion of the cluster is deduced.

\section{Observations and photometric reduction}

Three first epoch plates were taken with the $40 \mathrm{~cm}$ refractor $(f=6895 \mathrm{~mm})$ at Sheshan station of Shanghai Astronomical Observatory in 1958 (see Table 1). All of these were taken without filter but are roughly in the $B$ passband. Perhaps due to weather changes during the observations, the number of detectable stars on the plates varies significantly, although the exposure times of these plates are not very different. Altogether, there are 115 different stars measured on the three plates.

All plates were scanned using the PDS-1010MS instrument of Purple Mountain Observatory, Chinese Academy
Table 1. The three first-epoch plates taken with $40 \mathrm{~cm}$ refractor at sheshan station of Shanghai Observatory

\begin{tabular}{lccc}
\hline Plate No. & Observation date & Exposure & No. Stars \\
\hline CL 58001 & $1958-03-29$ & $60^{\mathrm{m}}$ & 38 \\
CL 58003 & $1958-02-13$ & $45^{\mathrm{m}}$ & 24 \\
CL 58004 & $1958-02-13$ & $60^{\mathrm{m}}$ & 112 \\
\hline
\end{tabular}

Table 2. CCD Observational material taken with the Vainu Bappu Telescope

\begin{tabular}{cccc}
\hline Frame & Observation date & Passband & Exposure $(\mathrm{s})$ \\
\hline $24 \mathrm{~m} 4147 \mathrm{i} 27 \mathrm{f}$ & $1996-03-24$ & $I$ & 60 \\
$24 \mathrm{~m} 4147 \mathrm{i} 28 \mathrm{f}$ & $1996-03-24$ & $I$ & 180 \\
$24 \mathrm{~m} 4147 \mathrm{i} 29 \mathrm{f}$ & $1996-03-24$ & $I$ & 900 \\
$24 \mathrm{~m} 4147 \mathrm{r} 30 \mathrm{f}$ & $1996-03-24$ & $R$ & 60 \\
$24 \mathrm{~m} 4147 \mathrm{r} 31 \mathrm{f}$ & $1996-03-24$ & $R$ & 180 \\
$24 \mathrm{~m} 4147 \mathrm{r} 32 \mathrm{f}$ & $1996-03-24$ & $R$ & 900 \\
$24 \mathrm{~m} 4147 \mathrm{~b} 33 \mathrm{f}$ & $1996-03-24$ & $B$ & 180 \\
$24 \mathrm{~m} 4147 \mathrm{~b} 34 \mathrm{f}$ & $1996-03-24$ & $B$ & 300 \\
$24 \mathrm{~m} 4147 \mathrm{~b} 35 \mathrm{f}$ & $1996-03-24$ & $B$ & 1800 \\
$24 \mathrm{~m} 4147 \mathrm{v} 36 \mathrm{f}$ & $1996-03-24$ & $V$ & 60 \\
$24 \mathrm{~m} 4147 \mathrm{v} 37 \mathrm{f}$ & $1996-03-24$ & $V$ & 180 \\
$24 \mathrm{~m} 4147 \mathrm{v} 38 \mathrm{f}$ & $1996-03-24$ & $V$ & 1200 \\
$24 \mathrm{~m} 4147 \mathrm{~b} 30 \mathrm{f}$ & $1996-03-25$ & $B$ & 1800 \\
$24 \mathrm{~m} 4147 \mathrm{v} 31 \mathrm{f}$ & $1996-03-25$ & $V$ & 1200 \\
$24 \mathrm{~m} 4147 \mathrm{r} 32 \mathrm{f}$ & $1996-03-25$ & $R$ & 900 \\
$24 \mathrm{~m} 4147 \mathrm{i} 33 \mathrm{f}$ & $1996-03-25$ & $I$ & 900 \\
\hline
\end{tabular}

of Sciences. Window scanning was used, with the initial coordinates converted from the CCD data (see below). The second epoch data in this paper are CCD frames taken in 1996 with 2.34 m Vainu Bappu telescope at Kavalur, India (focal length $7558 \mathrm{~mm}$, geographic position: E121 $11^{\prime} 11.3^{\prime \prime}, \mathrm{N} 31^{\circ} 05^{\prime} 47.6^{\prime \prime}$, altitude $713 \mathrm{~m}$ ). Table 2 gives the detailed observing information. The main purpose of taking these frames is to do $B V R I$ photometry, but they are also of interest for astrometry. Of these, four $B$-passband frames were used for the proper motion determination. The CCD was made by Photometrics Inc., type TK1024AB2, $1024 \times 1024$ pixels, each $24 \mu \times 24 \mu$, the field is $11^{\prime} \times 11^{\prime}$. This field size defines the investigated region in our work. Image processing of the CCD data frames was done in the usual manner using bias subtraction and flat-fielding. The coincidence of the flat field frames (summed for each colour band) is better than a few percent in all the filters. The magnitudes were determined using DAOPHOT2 and ALLSTAR2 profile fitting softwares (Stetson 1987, 1992). The stellar point spread function (PSF) was evaluated using the Penny model of DAOPHOT2 from several uncontaminated stars present in each frame. The image parameters and errors 
Table 3. Coefficients of linear transformation of $3 B$-passband CCD frames to the standard CCD frame (24m4147b33f)

\begin{tabular}{crrrrrr}
\hline \multirow{2}{*}{ Frame } & \multicolumn{3}{c}{$X$ coordinate } & \multicolumn{3}{c}{$Y$ coordinate } \\
\cline { 2 - 7 } & $a$ & $b$ & $c$ & $a^{\prime}$ & $b^{\prime}$ & $c^{\prime}$ \\
\hline 24m4147b34f & 1.00 & 0.00 & 0.38 & 0.00 & 1.00 & 0.82 \\
24m4147b35f & 1.00 & 0.00 & -0.31 & 0.00 & 1.00 & 1.49 \\
24m4147b30f & 1.00 & 0.00 & 16.42 & 0.00 & 1.00 & -21.80 \\
\hline
\end{tabular}

Note: $x^{\prime}=a x+b y+c, y^{\prime}=a^{\prime} x+b^{\prime} y+c^{\prime}$, in units of pixel.

Table 4. Average positional accuracy of the position of one star on the $B$-passband CCD frames (unit: pixel)

\begin{tabular}{cccc}
\hline Frame & $\sigma_{x}$ & $\sigma_{y}$ & star number \\
\hline 24m4147b33f & 0.22 & 0.20 & 106 \\
24m4147b34f & 0.18 & 0.17 & 103 \\
24m4147b35f & 0.20 & 0.19 & 104 \\
24m4147b30f & 0.21 & 0.16 & 101 \\
\hline
\end{tabular}

provided by DAOPHOT2 and ALLSTAR2 were used to reject poor measurements. About $10 \%$ of the stars were rejected in this process. After all the frames were reduced, Stetson's (1992) DAOMATCH and DAOMASTER routines were used to cross identify the stars measured on different frames of the cluster region.

In order to estimate the astrometric accuracy of the four $B$-passband CCD frames, we took the first frame $(24 \mathrm{~m} 4147 \mathrm{~b} 33 \mathrm{f})$ as a standard frame. Rectangular stellar positions (in units of pixel) in the other three $B$-passband frames were linearly transformed to the same system as the standard frame (coefficients of the transformation are listed in Table 3). The few stellar positions in those three frames that have significant coordinate deviations (exceeding 2 pixels) in comparison with the standard frame (probably due to erroneous identification or severe image blending) were rejected before further reductions. Then we obtained an average frame in which each stellar position is the mean of the $(x, y)$ coordinate in the four frames (in the same system). Coordinate deviations for stars in all four frames with respect to the average frame were calculated and stars with $x$ or $y$ deviations larger than 0.7 pixel were rejected. The resulting $(x, y)$ coordinate dispersion for the remaining stars is about 0.2 pixel, corresponding to 0 "'13 (see Table 4). We only used the remaining stars for further astrometric reductions (Table 4 also lists the number of stars used in each frame).

The CCD instrumental magnitudes have been calibrated using local standards in the cluster field by Christian et al. (1985). The equations relating the instrumental magnitudes to standard magnitudes are:

$B=b-(0.3213 \pm 0.0165)(b-v)$,

$V=v-(0.1528 \pm 0.0118)(v-i)$,
Table 5. Photometric errors (output of DAOPHOT) from the fit to the star profile

\begin{tabular}{ccc}
\hline Parameter & Median error & Maximum error \\
\hline$V$ & 0.025 & 0.296 \\
$B-V$ & 0.043 & 0.404 \\
$V-R$ & 0.045 & 0.301 \\
$V-I$ & 0.043 & 0.303 \\
\hline
\end{tabular}

$R=r+(0.0738 \pm 0.0287)(v-r)$,

$I=i+(0.0706 \pm 0.0156)(v-i)$,

where $b, v, r, i$ represent instrumental magnitudes while $B, V, R, I$ represent standard magnitudes.

The limiting magnitude of the photometry is close to $V=22 \mathrm{mag}$, in this paper however, only stars with proper motion data available were considered, whose limiting magnitude is about $B=17.6$. Table 5 shows the photometric error distribution for the 115 stars. The complete photometric work will be given in a separate paper by A.C. Gupta.

\section{Proper motion reduction and membership probability estimation}

For the reduction of the proper motions we used the catalogue of positions and proper motions of 82 stars based on Hipparcos system given by Geffert et al. (1997) reduced from positions of stars on plates taken with the $30 \mathrm{~cm}$ refractor $(f=5130 \mathrm{~mm})$ over 60 years (Brosche et al. 1985). The catalogue is in the J2000 system with an epoch of 1950. It has 54 stars in common with the present work. These stars served as reference stars. A Central overlapping procedure (Russell 1976; Wang et al. 1996) was used for the proper motion reduction. The four $B$ passband second epoch CCD frames were treated in the same way as the three first epoch plates and all will be designated as "plates" hereafter. The $x, y$ coordinates of the stellar images in the plates with units of CCD pixel were multiplied by a suitable factor in order to convert them into numbers in units of $\mathrm{mm}$, which are close to the units of the measurements of the first epoch plates. All of the 54 reference stars were adjusted in each iteration of position and proper motion reduction.

In the reduction we checked the need for different plate constants. It is shown that six plate constants would be enough for our central overlap procedure. Thus, higher order polynomials of the positions and additional magnitude and color terms were not necessary. Figure 1 gives the residuals in $x$ and $y$ with respect to magnitude and color, respectively, for plate CL 58004 (here we selected only stars which were seen on at least two of the first epoch plates), Fig. 2 gives the same relations for CCD 

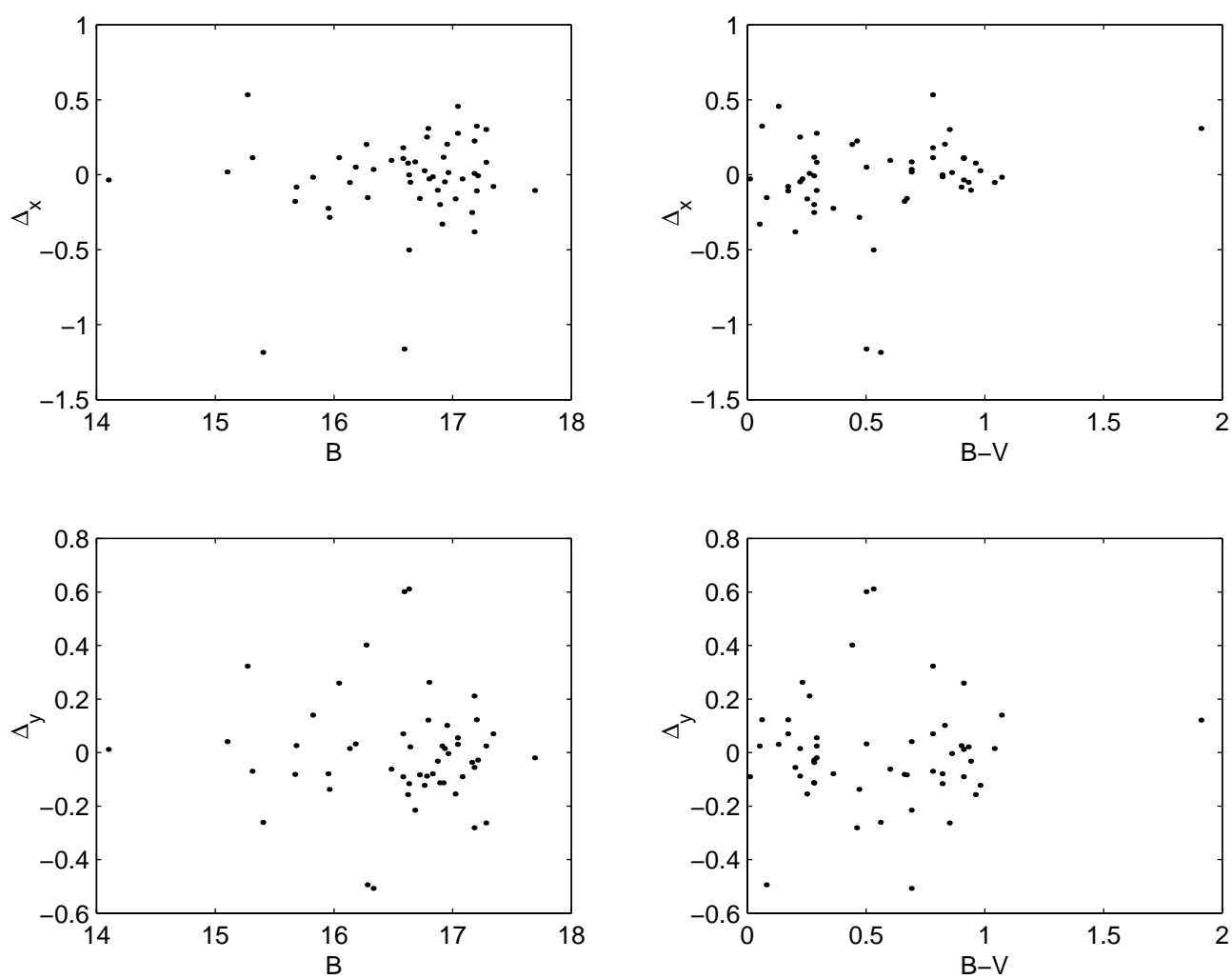

Fig. 1. Plate CL 58004: residuals in $X$ and $Y$ vs. mag and color
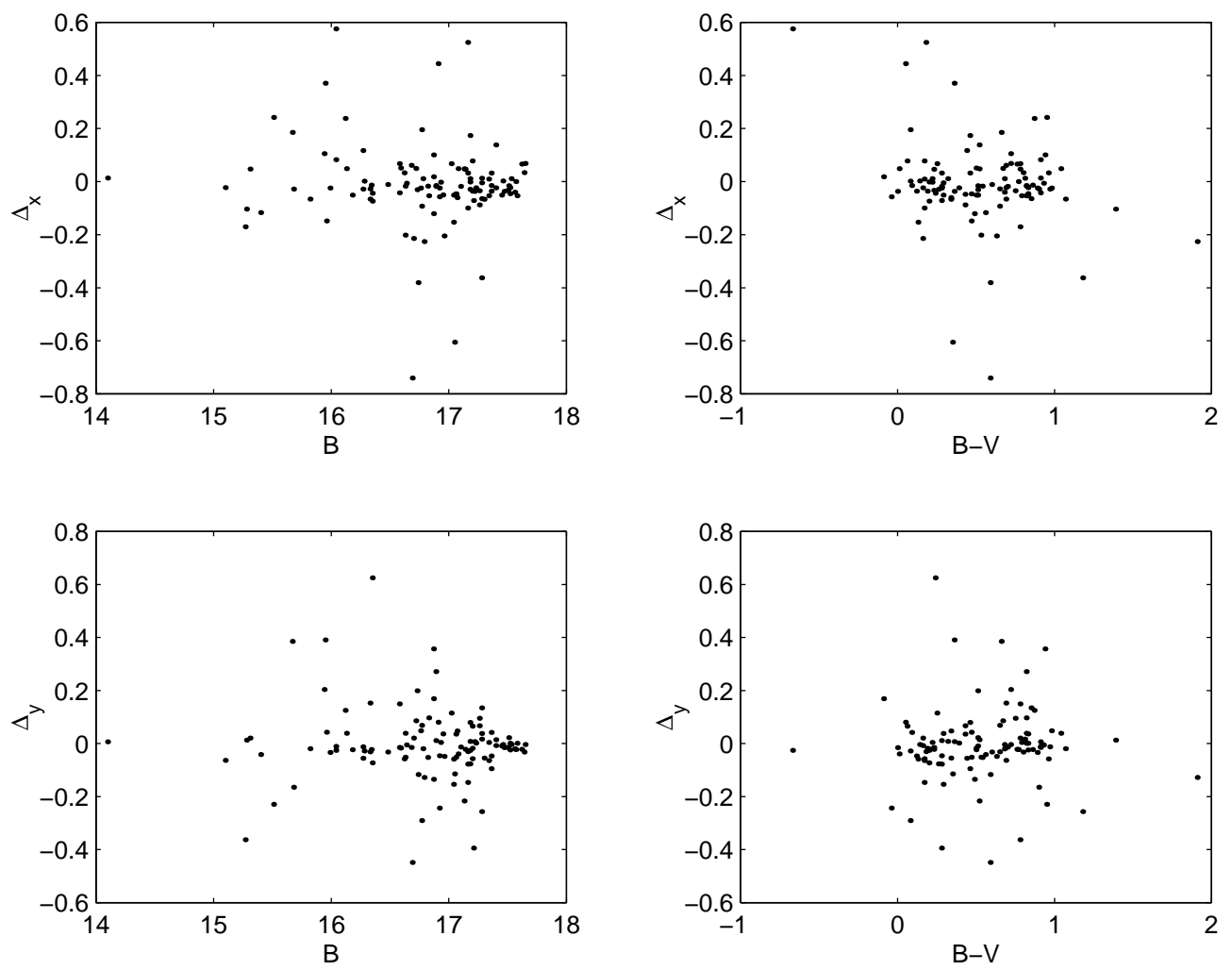

Fig. 2. CCD frame: residuals in $X$ and $Y$ vs. mag and color 

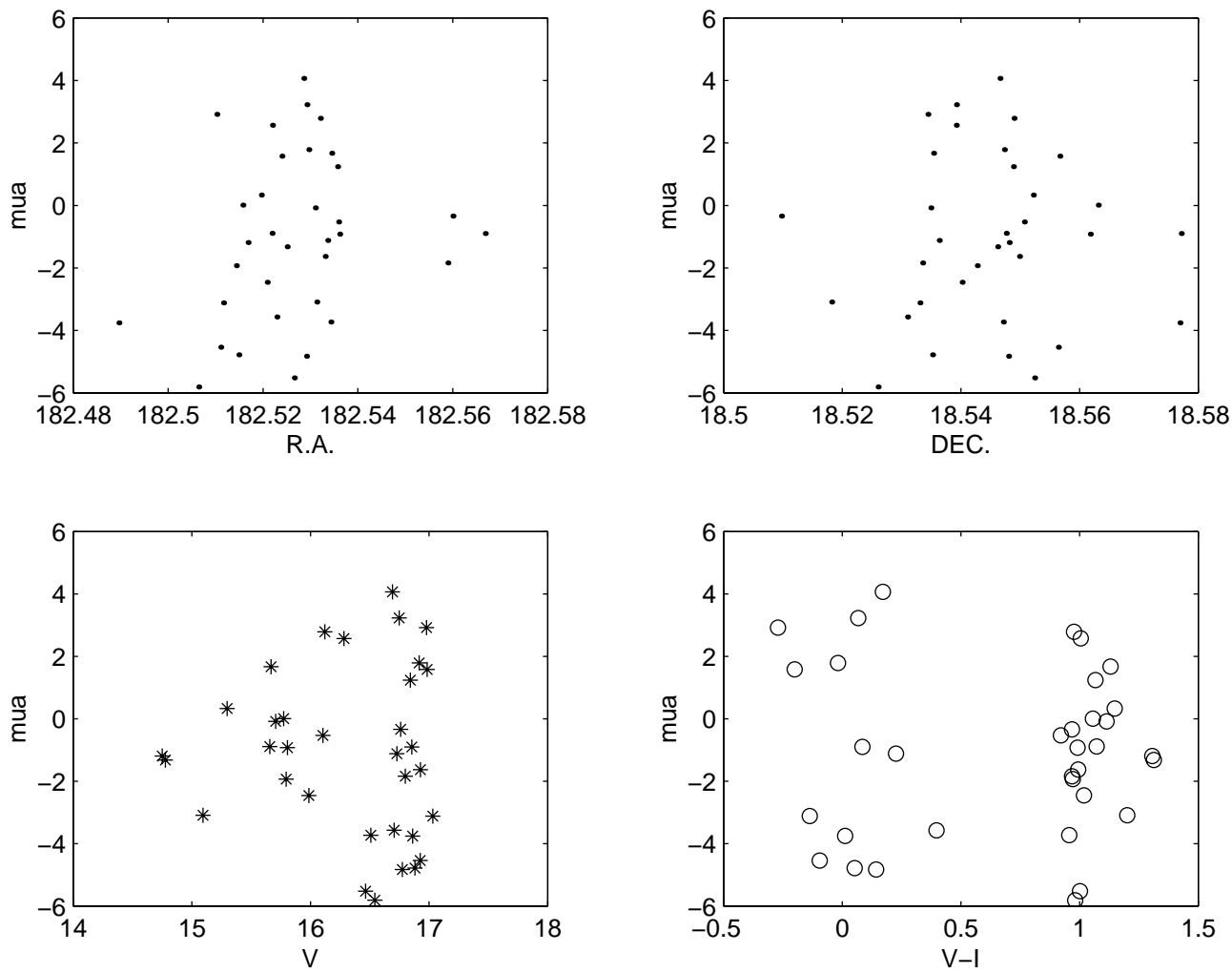

Fig. 3. $\mu_{\alpha} \cos \delta$ vs. RA, DEC., mag and color
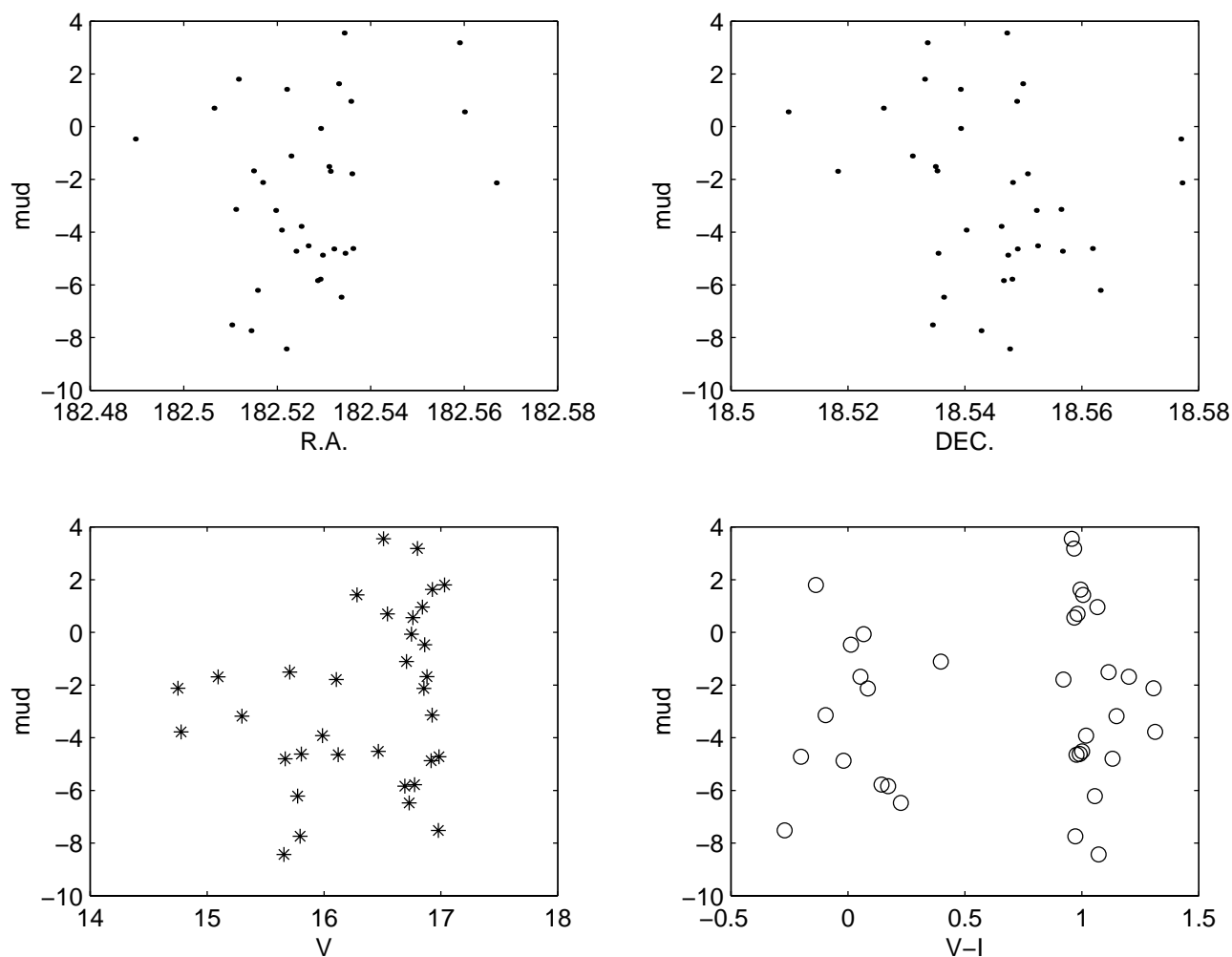

Fig. 4. $\mu_{\delta}$ vs. RA, DEC., mag and color 
Table 6. Internal astrometric errors

\begin{tabular}{ccc}
\hline Parameter & Median error & Maximum error \\
\hline$\alpha$ & 0.0041 & 0.0394 \\
$\delta$ & $0^{\prime \prime} .059$ & $0^{\prime \prime} .404$ \\
$\mu_{\alpha} \cos \delta$ & $1.45 \mathrm{mas} / \mathrm{yr}$ & $15.13 \mathrm{mas} / \mathrm{yr}$ \\
$\mu_{\delta}$ & $1.39 \mathrm{mas} / \mathrm{yr}$ & $11.88 \mathrm{mas} / \mathrm{yr}$ \\
\hline
\end{tabular}

frame 25m4147b30f. Both figures show no significant systematic trend. From these two figures one can find that the positional accuracy of the CCD frames is about 0 ". 13 , which is consistent with our estimation above, while the positional accuracy for photographic plates is about 0 ". 20 (after rejecting several stars with large deviations).

After the fourth iteration positions and proper motions from two subsequent iteration steps showed little deviations. Mean differences in position were smaller than 0 ".03, the rms is smaller than 6 mas and the differences in proper motion were below 1 mas/yr, the rms below $0.15 \mathrm{mas} / \mathrm{yr}$. We took the positions and proper motions of 115 stars given by this iteration as the final outcome and their errors are listed in Table 6 . It should be pointed out that the errors given in Table 6 are only estimates of internal errors in the adjustment process and are undoubtedly underestimated to some extent. In our following discussion on the membership probabilities, we shall give more realistic error estimates.

To search for possible systematic errors in our resulting proper motions, we selected a test sample of 34 stars with membership probabilities greater than 0.7 and of horizontal branch or the giant branch nature from the $V$ versus $(V-I)$ diagram (see Fig. 6 and text below). From this sample, the resulting proper motions were plotted against R.A., DEC., $V$ and $V-I$, respectively, as is shown in Fig. 3 and Fig. 4. No apparent systematic dependence could be found in these figures. However, as is seen in these figures, the proper motion dispersions are much greater than the internal errors in Table 6. The proper motion dispersions here are $2.7 \mathrm{mas} / \mathrm{yr}$ in right ascension and $3.2 \mathrm{mas} / \mathrm{yr}$ in declination, respectively, which should be related to the astrometric accuracy of the CCD frames and the photographic plates. For those stars which are seen on all three plates and four CCD frames, the accuracy is rather $3 \mathrm{mas} / \mathrm{yr}$.

For the membership probability estimation, we used a maximum likelihood method with a 9-parameter Gaussian model as follows:

$$
\begin{aligned}
\Phi\left(\mu_{x i}, \mu_{y i}\right)= & \Phi_{\mathrm{c}}\left(\mu_{x i}, \mu_{y i}\right)+\Phi_{\mathrm{f}}\left(\mu_{x i}, \mu_{y i}\right), \\
\Phi_{\mathrm{c}}\left(\mu_{x i}, \mu_{y i}\right)= & \frac{n_{\mathrm{c}}}{2 \pi\left(\sigma_{0}^{2}+\varepsilon_{x i}^{2}\right)^{1 / 2}\left(\sigma_{0}^{2}+\varepsilon_{y i}^{2}\right)^{1 / 2}} \\
& \cdot \exp \left\{-\frac{1}{2}\left[\frac{\left(\mu_{x i}-\mu_{x_{\mathrm{c}}}\right)^{2}}{\sigma_{0}^{2}+\varepsilon_{x i}^{2}}+\frac{\left(\mu_{y i}-\mu_{y_{\mathrm{c}}}\right)^{2}}{\sigma_{0}^{2}+\varepsilon_{y i}^{2}}\right]\right\},
\end{aligned}
$$

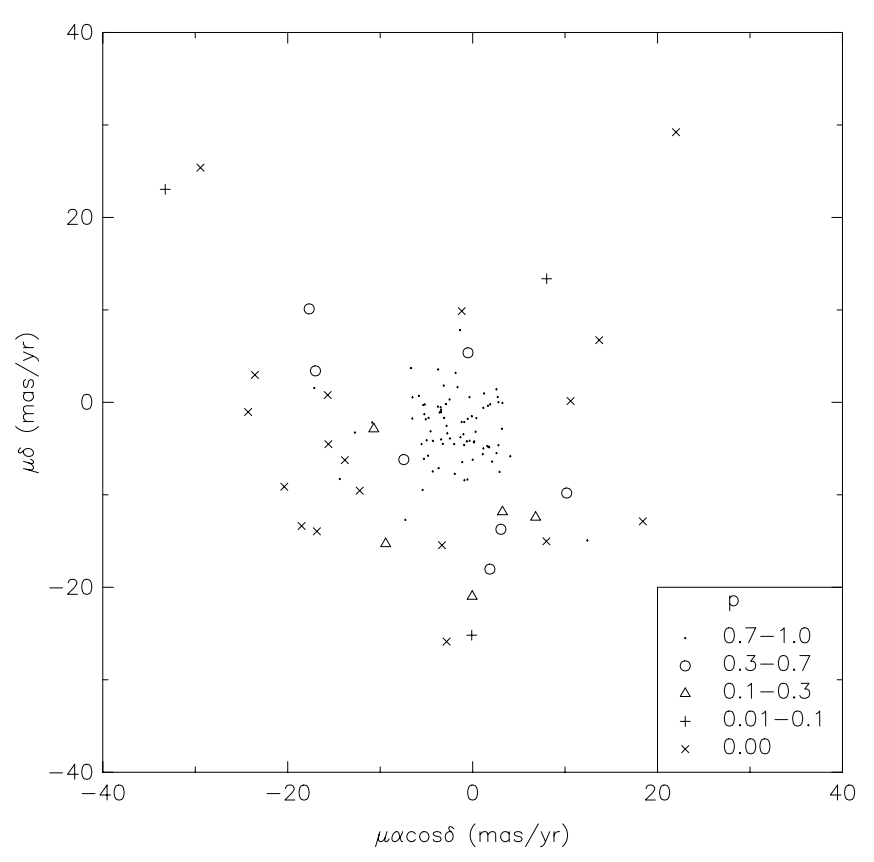

Fig. 5. Vector point diagram of proper motions

$$
\begin{aligned}
\Phi_{\mathrm{f}}\left(\mu_{x i}, \mu_{y i}\right)= & \frac{1-n_{\mathrm{c}}}{2 \pi\left(1-\rho^{2}\right)^{1 / 2}\left(\sigma_{x 0}^{2}+\varepsilon_{x i}^{2}\right)^{1 / 2}\left(\sigma_{y 0}^{2}+\varepsilon_{y i}^{2}\right)^{1 / 2}} \\
& \cdot \exp \left\{-\frac{1}{2\left(1-\rho^{2}\right)}\left[\frac{\left(\mu_{x i}-\mu_{x \mathrm{f}}\right)^{2}}{\sigma_{x 0}^{2}+\varepsilon_{x i}^{2}}\right.\right. \\
& -\frac{2 \rho\left(\mu_{x i}-\mu_{x \mathrm{f}}\right)\left(\mu_{y i}-\mu_{y \mathrm{f}}\right)}{\left(\sigma_{x 0}^{2}+\varepsilon_{x i}^{2}\right)^{1 / 2}\left(\sigma_{y 0}^{2}+\varepsilon_{y i}^{2}\right)^{1 / 2}} \\
& \left.\left.+\frac{\left(\mu_{y i}-\mu_{y f}\right)^{2}}{\sigma_{y 0}^{2}+\varepsilon_{y i}^{2}}\right]\right\} .
\end{aligned}
$$

where $\left(\mu_{x i}, \mu_{y i}\right)$ and $\left(\varepsilon_{x i}, \varepsilon_{y i}\right)$ are the proper motion components of the $i$-th star and their observing errors, respectively. The centers of the cluster and of the field stars in the proper motion vector point diagram, $\left(\mu_{x \mathrm{c}}, \mu_{y \mathrm{c}}\right)$ and $\left(\mu_{x \mathrm{f}}, \mu_{y \mathrm{f}}\right)$, the intrinsic dispersions of the proper motions of the cluster members and field stars $\sigma_{0}$ and $\left(\sigma_{x 0}, \sigma_{y 0}\right)$, the correlation coefficient $\rho$, and $n_{\mathrm{c}}$, the fraction of all member stars, have to be determined in the fit. The 9 parameters were determined by the maximum likelihood procedure and their uncertainties by the second derivative of the likelihood function. The results are summarized in Table 7. Once these parameters are determined, the membership probability of $i$-th star is given by

$p_{i}=\Phi_{\mathrm{c}}\left(\mu_{x i}, \mu_{y i}\right) / \Phi\left(\mu_{x i}, \mu_{y i}\right)$.

Figure 5 gives the vector point plot diagram of the 115 stars, with different symbols for different probabilities. The number of stars with different membership probabilities is shown in Table 8 . According to the value of $n_{\mathrm{c}}$ in Table 7 , there should be 70 cluster stars, under the assumption that stars with $p \leq 0.6$ can be regarded as field stars. 
Table 7. Estimates of model parameters from maximum likelihood fitting

\begin{tabular}{cr}
\hline Parameter & \multicolumn{1}{c}{ Estimate } \\
\hline$\mu_{x \mathrm{c}}$ & $-1.65 \pm 0.39 \mathrm{mas} / \mathrm{yr}$ \\
$\mu_{y \mathrm{c}}$ & $-2.69 \pm 0.38 \mathrm{mas} / \mathrm{yr}$ \\
$\mu_{x \mathrm{f}}$ & $-5.30 \pm 0.66 \mathrm{mas} / \mathrm{yr}$ \\
$\mu_{y \mathrm{f}}$ & $-4.13 \pm 2.63 \mathrm{mas} / \mathrm{yr}$ \\
$\sigma_{\mathrm{o}}$ & $2.58 \pm 0.33 \mathrm{mas} / \mathrm{yr}$ \\
$\sigma_{x_{\mathrm{o}}}$ & $12.69 \pm 0.10 \mathrm{mas} / \mathrm{yr}$ \\
$\sigma_{y_{\mathrm{o}}}$ & $11.52 \pm 1.40 \mathrm{mas} / \mathrm{yr}$ \\
$\rho$ & $-0.105 \pm 0.095$ \\
$n_{\mathrm{c}}$ & $0.698 \pm 0.051$ \\
\hline
\end{tabular}

Table 8. Membership probability distribution of stars in the region of NGC 4147

\begin{tabular}{cc}
\hline Prob. & Number of stars \\
\hline $1.00-0.90$ & 59 \\
$0.90-0.80$ & 16 \\
$0.80-0.70$ & 6 \\
$0.70-0.60$ & 2 \\
$0.60-0.50$ & 2 \\
$0.50-0.40$ & 2 \\
$0.40-0.30$ & 1 \\
$0.30-0.20$ & 2 \\
$0.20-0.10$ & 3 \\
$0.10-0.01$ & 3 \\
0.00 & 19 \\
\hline
\end{tabular}

The values in Table 7 provide a possibility of estimating the external accuracy of the proper motion results in this work. Since NGC 4147 is very far away from us the contribution by the internal motions of the cluster stars can be neglected and $\sigma_{0}$ can be regarded entirely as the observational dispersion. The dispersion of $2.6 \mathrm{mas} / \mathrm{yr}$ is in good agreement with the error estimated from the astrometric accuracy of the CCD frames and the plates.

\section{Color-magnitude diagram}

The color-magnitude diagrams of $V$ versus $B-V, V-R$ and $V-I$ are presented in Figs. 6-8, respectively, with different symbols for different membership probabilities as used in Fig. 5.

The characteristic behaviour of these diagrams is, on the whole, similar to the CMD of $V$ versus $B-V$ from Aurière \& Lauzeral (1991), however with a large dispersion for the cluster members, especially there are quite a few stars with large membership probabilities located above the horizontal branch. From our CMDs of $V$ versus $V-R$ and $V-I$, it seems that there exists a second horizontal branch (somewhat similar to the second

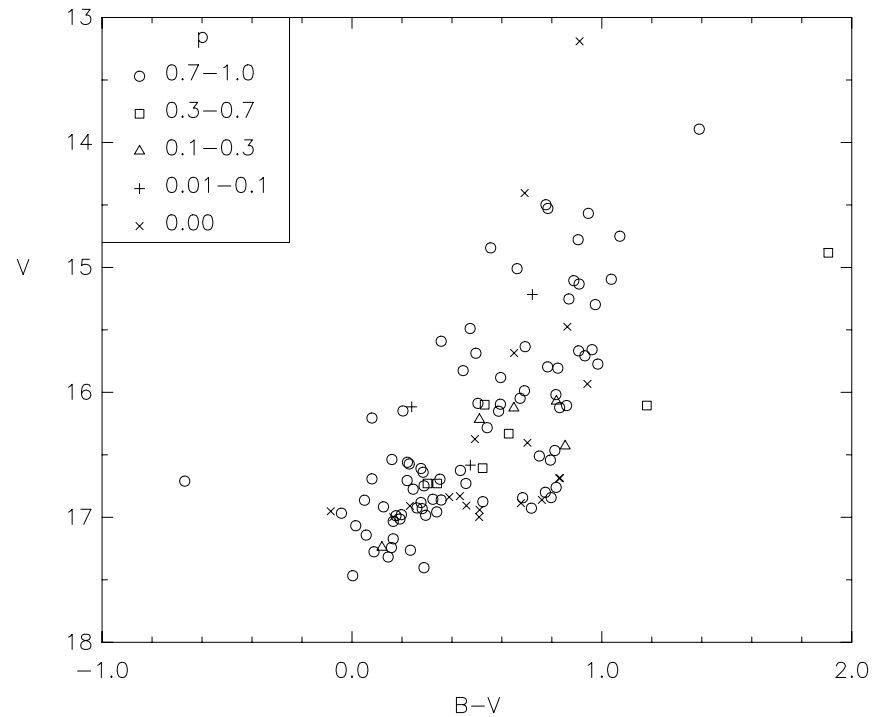

Fig. 6. Diagram of $V$ vs. $B-V$

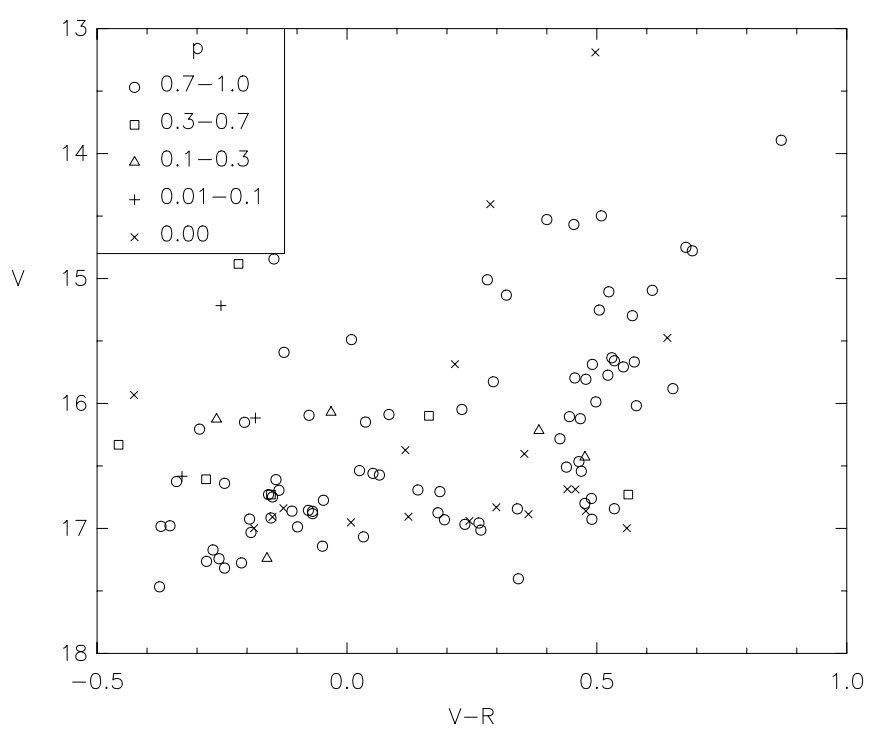

Fig. 7. Diagram of $V$ vs. $V-R$

main sequence in CMD of some open clusters). It might be caused by double stars. On the other hand in our sample, we have not identified the RR Lyrae stars which would drift off the horizontal branch. Moreover, crowding problems near the cluster center caused the reduced accuracy of the photometric data, and this could be another reason for the large dispersion in the member star sequence. In our sample 54 stars, which appeared only on one of the first-epoch plates (CL 58004) and not in the catalogue of Geffert et al. (1997), have relatively large uncertainties in the membership probabilities since their proper motion estimates can only be regarded as preliminary. In addition, some of our stars with large membership probabilities have large internal proper motion errors (which can be seen in the vector point diagram of proper motions). In this case, it is likely that one or two non-members were estimated 


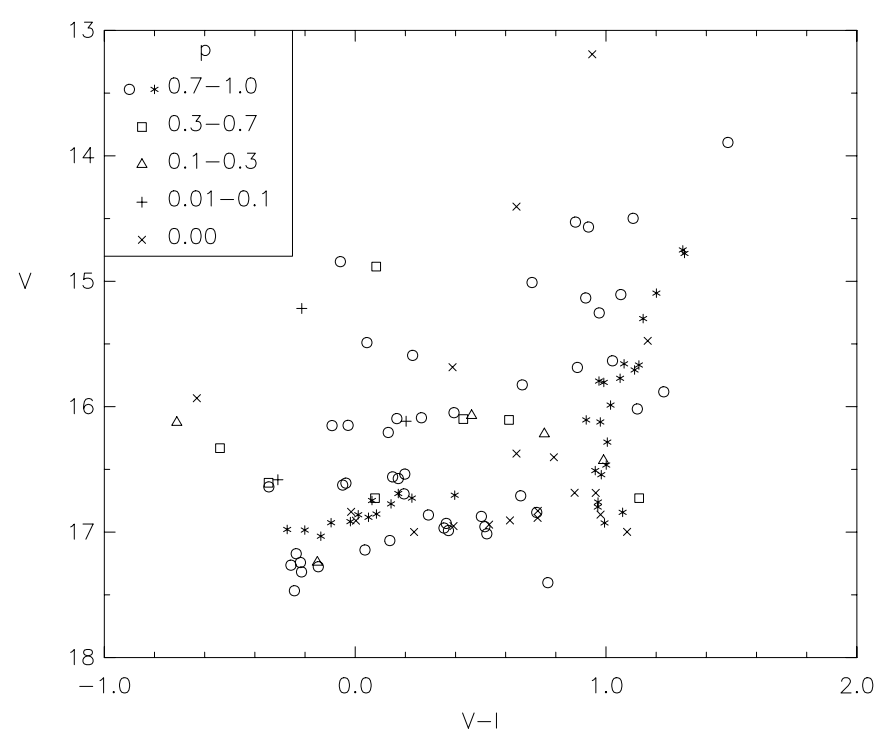

Fig. 8. Diagram of $V$ vs. $V-I,{ }^{*}$ : stars located on HB or GB and constitute a test sample

as member stars, which could also lead to an increase of the dispersion in the CMDs. It is interesting to note that the star sorted out by Aurière \& Lauzeral (1991), being brighter than the red giants by about 1 mag and redder by $0.4 \mathrm{mag}$ is, according to our result, a cluster member with a membership probability of 0.97 . It is located on the extension of the red giant branch in all three CMDs. Based on this, it is very probably an AGB star which is entering or has entered already the thermal pulsation stage. Since the cluster radial velocity is large, and the star not exceedingly faint, a future radial velocity measurement may check its membership.

\section{Illustration of the catalogue}

In Table 9 we give absolute proper motions as well as $B V R I$ photometric data for 115 stars in the region of globular cluster NGC 4147. The investigated region is about $11^{\prime} \times 11^{\prime}$ in size. The limiting magnitude of 115 stars which have proper motion data available is close to $B=17.6$ mag. The complete photometry on NGC 4147 will be given in a separate paper by A.C. Gupta. Detailed description about the observation, plate measurements and astrometric reduction can be found in Sect. 2 and Sect. 3 of this paper. In Table 9 the 115 stars were arranged in two parts. The first part includes 61 stars which appear on at least two of the first-epoch plates, or which have the position and proper motion data from Geffert et al. (1997) though it might appear on only one of their first-epoch plates. The second part includes 54 stars which appear on only one of our first-epoch plates, and these proper motions should be regarded as preliminary. The meaning of each column in Table 9 is as follows:

(1). sequence number of stars in this catalogue,
(2). sequence number in the complete photometric catalogue of A.C. Gupta,

(3). sequence number in Geffert et al. (1997),

(4). internal star ID number in Geffert et al. (1997),

(5). $V$ magnitude,

(6). standard error of the $V$ magnitude (in units of $0.001 \mathrm{mag})$,

(7). $B-V$ color index,

(8). standard error of $B-V$ (in units of $0.001 \mathrm{mag}$ ),

(9). $V-R$ color index,

(10). standard error of the $V-R$ (in units of $0.001 \mathrm{mag}$ ),

(11). $V-I$ color index,

(12). standard error of $V-I$ (in units of $0.001 \mathrm{mag}$ ),

(13). right ascension relative to J2000.0 equator and vernal equinox for epoch 2000 ,

(14). standard error of the right ascension (in units of $0.00001 \mathrm{~s})$,

(15). declination relative to J2000.0 equator and vernal equinox for epoch 2000,

(16). standard error of the declination (in units of 0 .'0001),

(17). absolute proper motion in right ascension (in units of $0.01 \mathrm{mas} / \mathrm{yr}$ ),

(18). standard error of the absolute proper motion in right ascension (in units of $0.01 \mathrm{mas} / \mathrm{yr}$ ),

(19). absolute proper motion in declination (in units of $0.01 \mathrm{mas} / \mathrm{yr}$ ),

(20). standard error of the absolute proper motion in declination (in units of $0.01 \mathrm{mas} / \mathrm{yr}$ ),

(21). number of plates contain this star,

(22). membership probability,

(23). member selection (0: non-member, 1: member).

\section{Proper motion, space velocity and orbital parameters of the cluster}

We have obtained three values for absolute proper motion of the cluster, from (1) the maximum likelihood fitting $(-1.65 \pm 0.39,-2.69 \pm 0.38) \mathrm{mas} / \mathrm{yr} ;(2)$ the mean value of proper motions of all stars, weighted by membership probabilities $(-2.08 \pm 0.41,-3.07 \pm 0.39)$ mas $/ y r ;(3)$ the mean value of proper motions of all members, equally weighted $(-1.94 \pm 0.47,-2.86 \pm 0.39)$ mas/yr. In comparison, Geffert et al. (1997) gave the value $(-1.0,-3.5) \mathrm{mas} / \mathrm{yr}$ (this value is also used by Odenkirchen et al. 1997). All these results can be regarded as consistent within the uncertainties. We take the second set as our final estimation of the absolute proper motion of the cluster. Taking into account a systematic error of the Hipparcos proper motions of $\pm 0.25 \mathrm{mas} / \mathrm{yr}$, we have the absolute proper motion of the cluster as $(-2.08 \pm 0.48,-3.07 \pm 0.46)$ mas/yr. We calculated the space velocity of NGC 4147 in a system of galactic standard of rest using a distance of the Sun from the galactic center of $8 \mathrm{kpc}$, a rotation of $225 \mathrm{~km} \mathrm{~s}^{-1}$ at the place of the Sun, 
Table 9. Absolute proper motions and BVRI photometry of 115 stars in the region of globular cluster NGC 4147

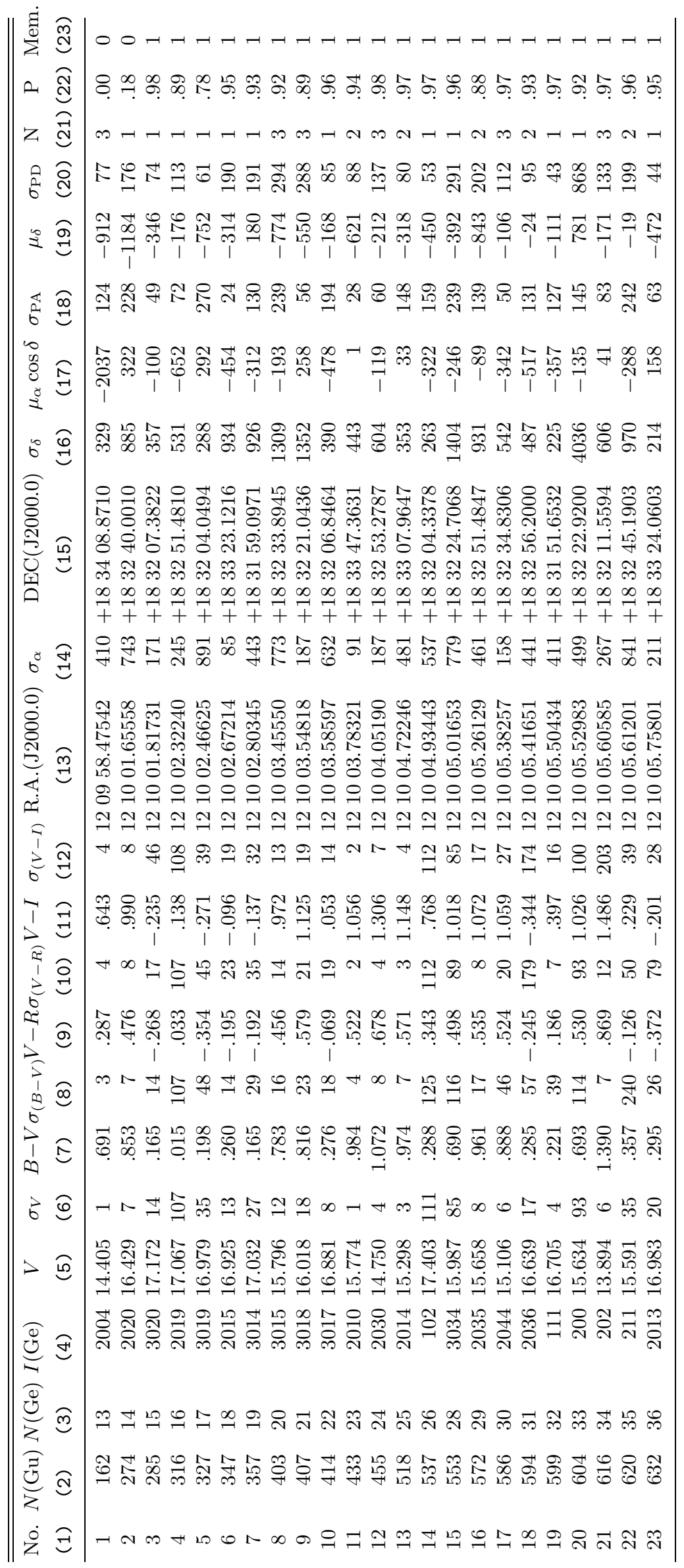


Table 9. continued

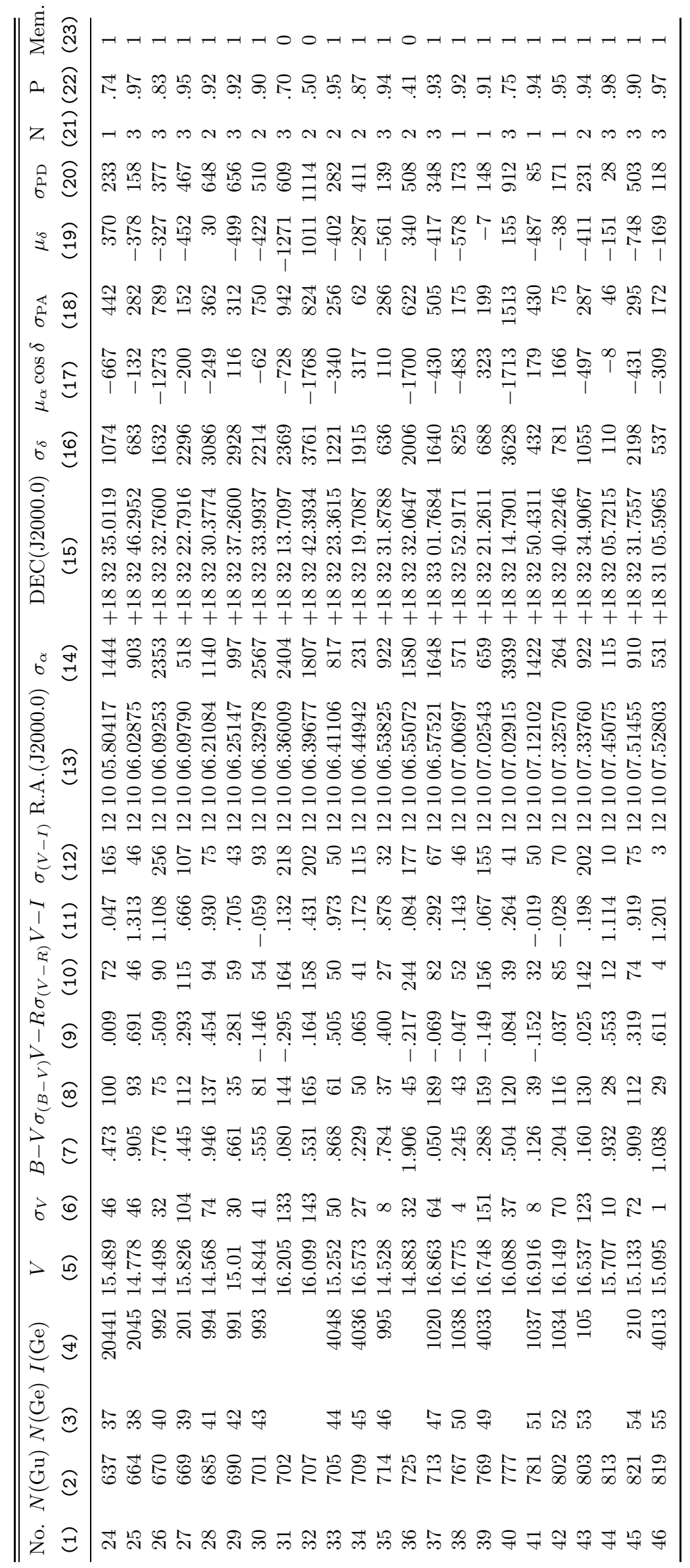


Table 9. continued

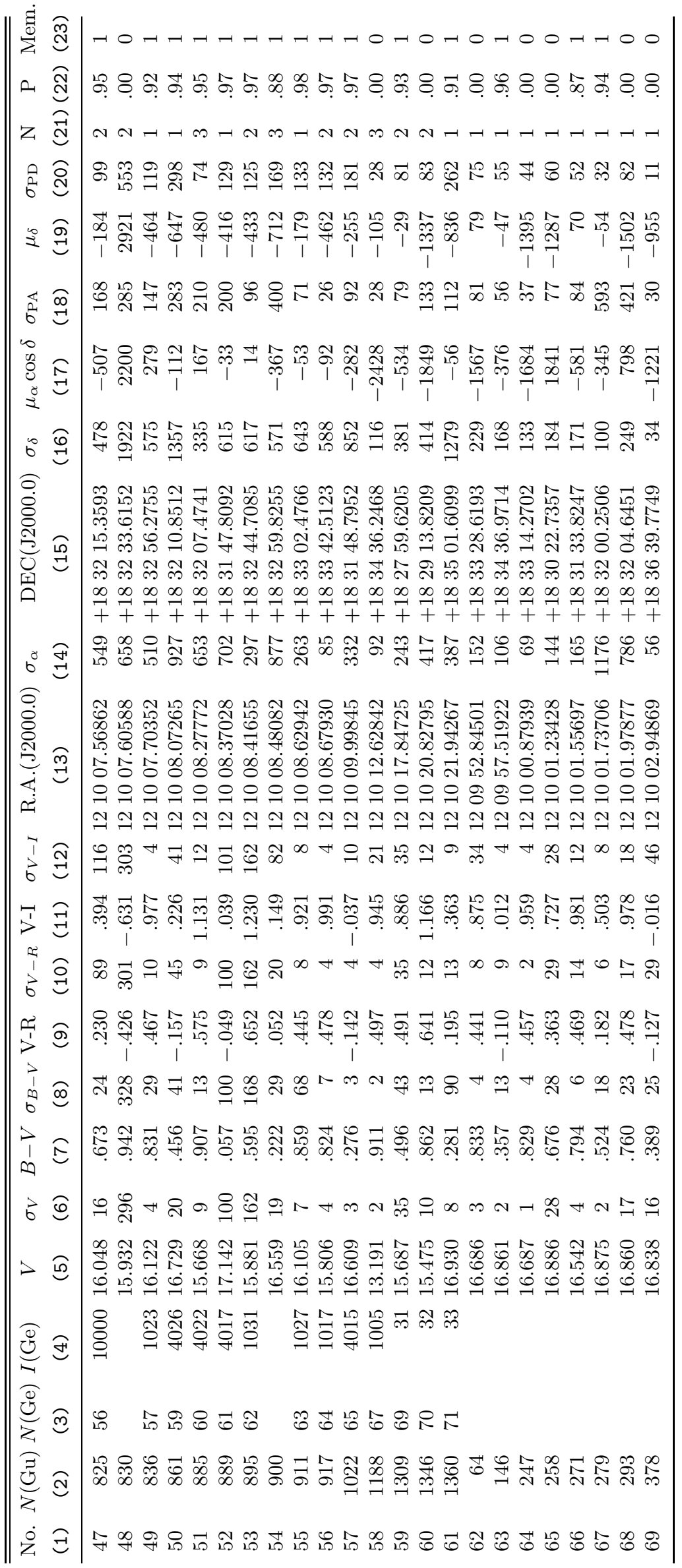


Table 9. continued

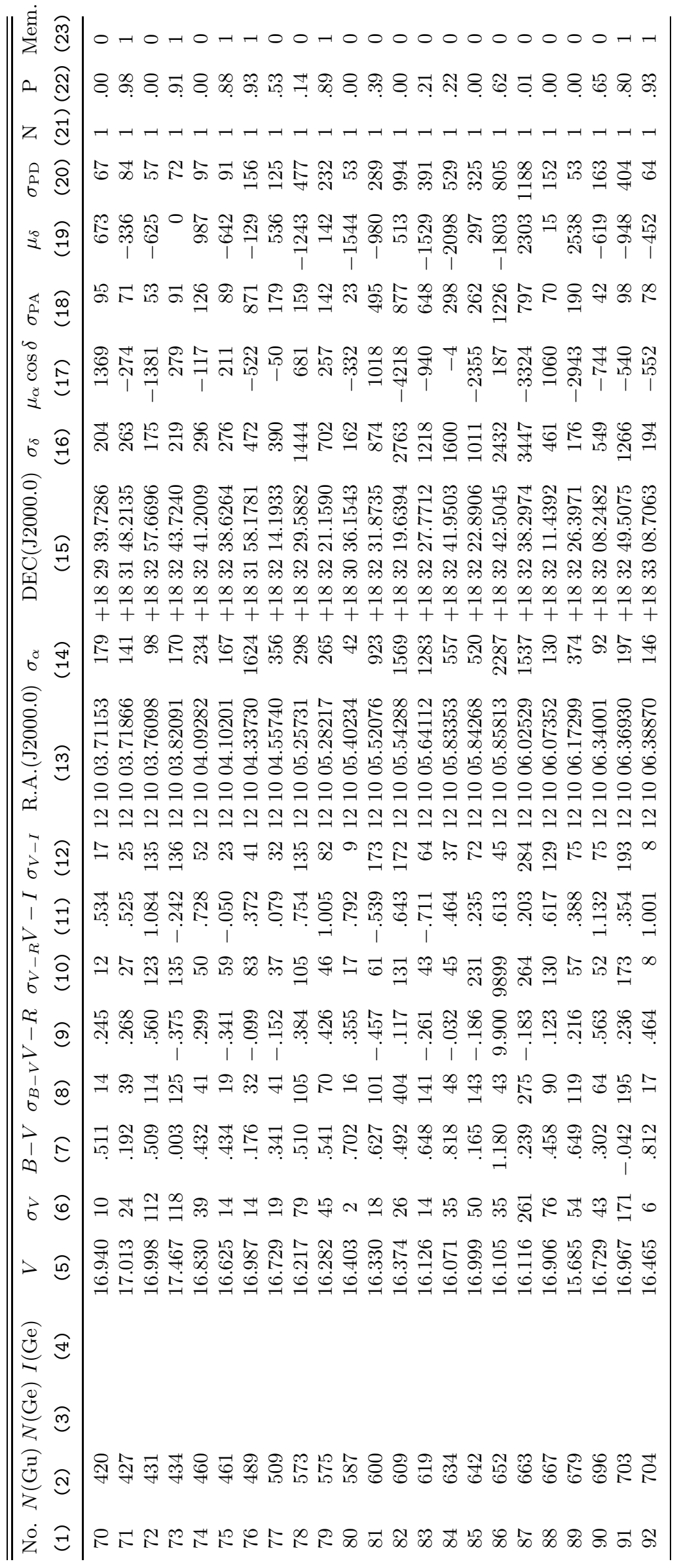


Table 9. continued

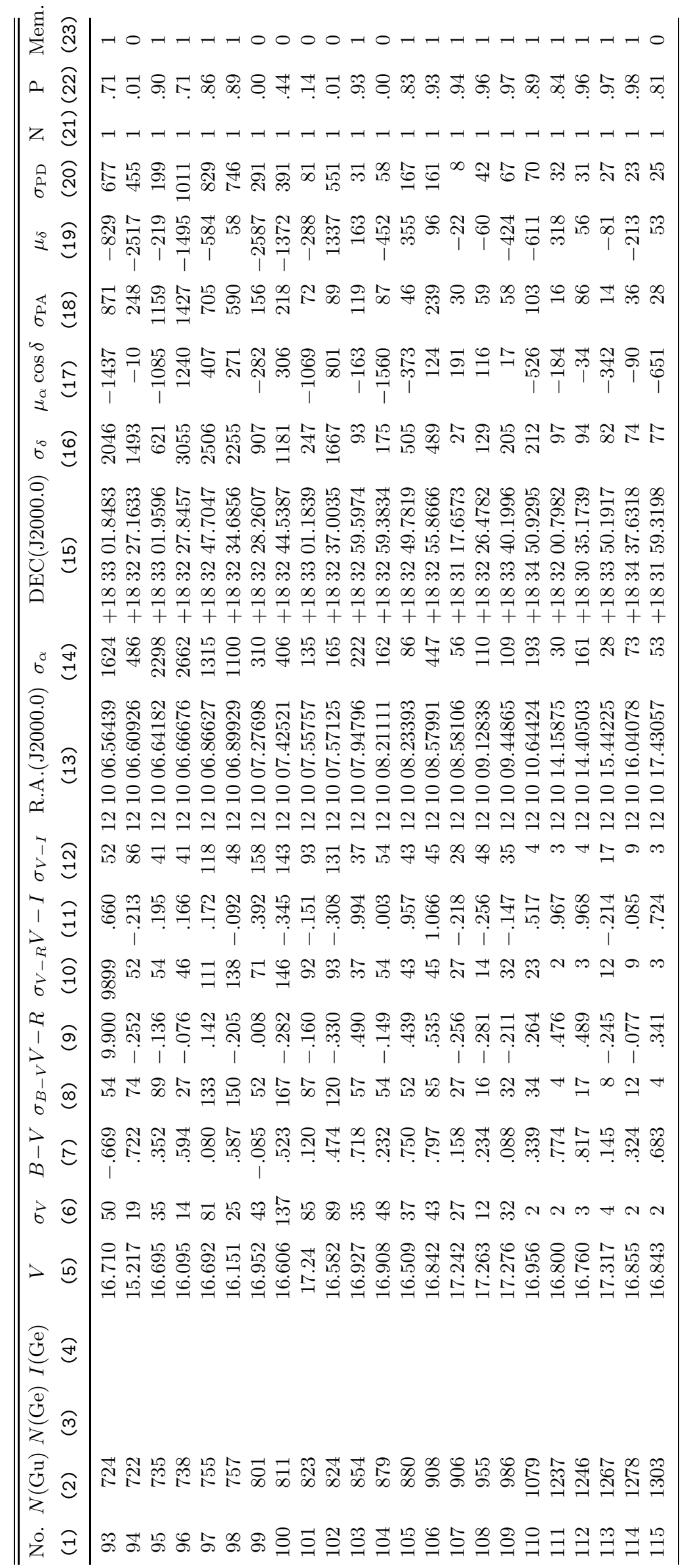


Table 10. Space motion and orbital parameters of NGC 4147

\begin{tabular}{crccccccc}
\hline PM value & $\begin{array}{r}\Pi \\
\left(\mathrm{km} \mathrm{s}^{-1}\right)\end{array}$ & $\begin{array}{c}\Theta \\
\left.\mathrm{km} \mathrm{s}^{-1}\right)\end{array}$ & $\begin{array}{c}Z \\
\left(\mathrm{~km} \mathrm{~s}^{-1}\right)\end{array}$ & $\begin{array}{c}R_{\mathrm{a}} \\
(\mathrm{kpc})\end{array}$ & $\begin{array}{c}R_{\mathrm{p}} \\
(\mathrm{kpc})\end{array}$ & & & \\
& & & & & \\
\hline$(2)$ & +70 & -91 & 116 & 22.5 & 4.2 & 0.69 & 89.2 \\
$(2)+1 \sigma$ & +104 & -58 & 123 & 22.0 & 2.3 & 0.81 & 84.2 \\
$(2)-1 \sigma$ & +26 & -124 & 109 & 23.5 & 6.9 & 0.55 & 91.7 \\
\hline
\end{tabular}

a peculiar velocity of the Sun relative to LSR of $U=10 \mathrm{~km} \mathrm{~s}^{-1}, V=15 \mathrm{~km} \mathrm{~s}^{-1}$ and $W=8 \mathrm{~km} \mathrm{~s}^{-1}$ (note that $U$ points from the Sun to the galactic center, $V$ is parallel to the galactic plane in the direction of the galactic rotation and $W$ perpendicular to the galactic plane), a heliocentric distance of NGC 4147 of $18.32 \mathrm{kpc}$ and a heliocentric radial velocity of NGC 4147 (from Harris 1996) of $183.2 \mathrm{~km} \mathrm{~s}^{-1}$. The resulting space velocity of NGC 4147 is $164 \pm 24 \mathrm{~km} \mathrm{~s}^{-1}$. Taking the proper motion value from just the maximum likelihood fitting, we obtained the space velocity as $142 \pm 12 \mathrm{~km} \mathrm{~s}^{-1}$, which is, within the uncertainty range, in a good agreement with the above.

We didn't take into account the uncertainty of the heliocentric distance determination of the cluster in the above reductions. Let us assume the adopted distance of the cluster has a relative error of $10 \%$. With this error assumption, we used two extreme distance values (that is, by adding $\pm 1 \sigma$ variations to the adopted one) as input data for a similar calculation. The derived space velocity values are $183 \pm 30 \mathrm{~km} \mathrm{~s}^{-1}$ and $149 \pm 18 \mathrm{~km} \mathrm{~s}^{-1}$, respectively, which are consistent with our adopted value of $164 \pm 24 \mathrm{~km} \mathrm{~s}^{-1}$ within the uncertainty range.

Therefore we take the space velocity result derived without considering the distance uncertainty as input value in the following kinematic discussions.

The velocity components of NGC 4147 in the galactic standard of rest as well as the orbital parameters calculated using the potential model from Dauphole \& Colin (1995) are listed in Table 10. In addition, we varied the cluster proper motion by $\pm 1 \sigma$ and re-calculated the corresponding velocity and orbital parameters, which are also listed in Table 10 . The velocity components $\Pi, \Theta, Z$ are in a system of galactic standard of rest. $\Pi$ points radially outwards from the galactic center to the cluster, $\Theta$ is in the direction of the galactic rotation, $Z$ is perpendicular to the galactic plane and towards the galactic north pole; $R_{\mathrm{a}}$ is the apogalactic distance, $R_{\mathrm{p}}$ is the perigalactic distance, $e$ is the eccentricity, and $i$ is the mean inclination angle of the orbit plane.

\section{Discussion}

The space velocity and the orbital parameters of NGC 4147 in Table 10 are quite different from those given by Dauphole \& Colin (1995), but are in good agreement with the values given by Odenkirchen et al. (1997). The major difference to the work of Dauphole \& Colin (1995) lies in the velocity component $\Theta$, where our values change the cluster's prograde rotation of Dauphole \& Colin (1995) into a retrograde motion. Since the inclination angle of the cluster orbit is close to 90 degree, small changes in the proper motion may change the sign of $\Theta$. Both of the absolute proper motion results from this paper and from Odenkirchen et al. (1997) are based on the Hipparcos reference frame and should have a better reliability. However, large proper motion errors of the single stars exist in the previous work while the proper motion reduced in the present work should be better due to deeper first epoch plates. From the relationship between apogalactic distance $R_{\mathrm{a}}$ and metallicity $[\mathrm{Fe} / \mathrm{H}]$ of 26 globular clusters, Dauphole et al. (1996) proposed the existence of a metallicity gradient among the halo globular clusters and they took this as a support to the rapid collapse model of the Galaxy (ELS). It seems that such a metallicity gradient is present in their Fig. 4. NGC 4147 is one of the two clusters, which do not fit very well to the proposed relation. As for NGC 4147, according to Dauphole \& Colin (1995), its apogalactic distance is $52 \mathrm{kpc}$, while its metallicity $[\mathrm{Fe} / \mathrm{H}]=-1.80$. If the metallicity gradient is true for the halo clusters, NGC 4147 should have an apogalactic distance smaller than 40 kpc. Using $R_{\mathrm{a}}$ calculated here or from Odenkirchen et al. (1997), the apogalactic distance of NGC 4147 will fit better to the apogalactic relation of distance and metallicity and NGC 4147 will be then a "normal" cluster. Among the smaller sample of 15 clusters with proper motions based on Hipparcos reference stars in Odenkirchen et al. (1997) the relation between $R_{\mathrm{a}}$ and $[\mathrm{Fe} / \mathrm{H}]$ is hardly seen. What can be inferred from their work is that the more metal-rich clusters are concentrated towards the galactic center and that the group of clusters with retrograde orbits (including NGC 4147) is in general chemically quite homogeneous, with $[\mathrm{Fe} / \mathrm{H}]$ between -1.5 to -2.0 . This conclusion is to a certain extent in favor of Searle \& Zinn's (1978) accretion model, but it not necessarily excludes the ELS model. Our results support the conclusion of Odenkirchen et al. (1997). Obviously more accurate absolute proper motion data of globular clusters should be obtained before we can make definite conclusion on the existence of the metallicity gradient and which of the ELS and SZ models would be the most favorable scenario for the formation of our Galaxy.

\section{References}

Allen C.W., 1973, Astrophysical Quantities, Third edition, University of London. The Athlone Press

Aurière M., Lauzeral C., 1991, A\&A 244, 303

Brosche P., Geffert M., Ninkovic S., 1983, Publ. Astron. Inst., Czech. Acad. Sci. 56, 145 
Brosche P., Geffert M., Klemola A.R., et al., 1985, AJ 90, 2033

Christian C.A., Adams M., Barnes J.V., et al., 1985, PASP 97, 363

Dauphole B., Geffert M., Colin J., Ducourant C., Odenkirchen M., Tucholke H.-J., 1995, A\&A 313, 119

Eggen O., Lynden-Bell D., Sandage A., 1962, ApJ 136, 762 (ELS)

Friel E.D., Heasly J.N., Christian C.A., 1987, PASP 99, 1248

Geffert M., Dauphole B., Colin J., et al., 1995, Stellar Populations, IAU Symp. 164. Kluwer Academic Publishers, p. 406

Geffert M., Hiesgen M., Colin J., Dauphole B., Ducourant C., 1997, in ESA SP-402. ESA Publications Division
Noordwijk, p. 579

Odenkirchen M., Brosche P., Geffert M., Tucholke H.-J., 1997, New Astron. 2, 477

Russell J.L., 1976, Ph.D. Thesis, University of Pittsburgh

Sandage A.R., Walker M.F., 1955, AJ 60, 230

Searle L., Zinn R., 1978, ApJ 225, 357 (SZ)

Stetson P.B., 1987, PASP 99, 191

Stetson P.B., 1992, IAU Coll. No. 136, "On Stellar Photometry - Current Techniques and Future Developments" Butler C.J. \& Elliot I. (eds.), p. 291

Wang J.J., Chen L., Zhao J.H., Jiang P.F., 1996, CA\&A 20, 364

Zhao J.H., Zhao J.L., 1994, Ann. Shang. Obs. 15, 85 